\title{
Estado Nutricional en Adolescentes de 17 Años y su Relación con su Historia de Caries. Florida 2011
}

\author{
Nutritional Status of 17-Year-Old Adolescents and \\ its Relation with Caries History. Florida 2011
}

\author{
Carlos Araya Vallespir"; Diana Marchant Obreque**; Pablo Rivas Delgado**; \\ Cristobal Palma Thomas ${ }^{*+1+k}$ \& Oscar Poblete de la Fuente
}

ARAYA, V. C.; MARCHANT, O. D.; RIVAS, D. P.; PALMA, T. C. \& POBLETE, F. O. Estado nutricional en adolescentes de 17 años y su relación con su historia de caries. Florida 2011. Int. J. Odontostomat., 8(2):171-176, 2014.

RESUMEN: Dado los altos índices de obesidad es necesario recopilar información actualizada para orientar nuevos programas preventivos, según el nivel de riesgo, en etapas más temprana del desarrollo humano y adolescencia, siendo éstos capaces de captar e integrar nuevos estilos de vida saludables. El objetivo fue evaluar el estado nutricional en adolescentes de 17 años de establecimientos educacionales de Florida y ver su relación con el índice COPD. Se realizó un estudio observacional descriptivo de diseño transversal. Se examinaron 86 estudiantes mediante una encuesta previamente validada, de recordatorio de ingesta alimenticia de las últimas 24 horas, la cual se aplicó en tres ocasiones, además se cuantificó el peso y la talla individual. Se obtuvieron datos acerca de ruralidad, urbanidad y sexo. Se aplicó un examen buco dentario, bajo las normas dela OMS, para determinar historia de caries (índice COPD). Se encontró un $2 \%$ de los jóvenes con bajo peso, $58 \%$ normales y $40 \%$ obesos, con una tasa de obesidad mayor en las mujeres, con diferencias significativas. En cuanto al origen urbano/rural se observa una distribución homogénea, donde las diferencias observadas no tuvieron significancia estadística. En relación al índice COPD, los hombres obtuvieron un 8,87 y las mujeres 9,28 , habiendo diferencias significativas entre ellos. No se encontró asociación estadística entre COPD y estado nutricional. Los jóvenes de 17 años de los establecimientos educacionales de Florida presentan un mal estado nutricional, reflejado en el alto porcentaje de obesos y un muy alto nivel de historia de caries, según la OMS.

PALABRAS CLAVE: adolescentes, historia de caries, nutrición.

\section{INTRODUCCIÓN}

La pubertad y adolescencia se caracterizan por un aumento de la velocidad de crecimiento, cambios en la composición corporal, aparición de caracteres sexuales secundarios y maduración emocional y psicosocial. Estas características influyen tanto en las recomendaciones alimentarias durante esta etapa de la vida, como en los hábitos alimentarios de los adolescentes (Gidding et al., 2006).

La autonomía e independencia propias de la edad, se acompañan de un aumento de las actividades fuera del hogar; con horarios de comida desordenados, omisiones de algún tiempo de alimentación (de- sayuno, almuerzo) y un aumento de la ingesta de comida rápida, golosinas y bebidas energéticas. Al mismo tiempo disminuye el consumo de lácteos, frutas y verduras; la cena puede pasar a ser la principal alimentación del día. El sedentarismo, sumado a estos hábitos alimentarios, contribuye a un riesgo elevado de obesidad y otras enfermedades crónicas (Larson et al., 2006; Niemeir et al., 2006).

Los estudios realizados en el país en los últimos años coinciden en destacar el bajo consumo de lácteos, verduras y frutas y el elevado consumo de productos de alta densidad energética en los niños.

\footnotetext{
Profesor Asociado Facultad de Odontología Universidad de Concepción.

EDF Hospital de Florida, Florida, Chile.

*** EDF Hospital de Cañete, Cañete, Chile.

**** EDF Vichuquén, Vichuquén, Chile.

**** Dentista Tratante, Santiago, Chile.

${ }^{* * * * * *}$ Departamento de Salud Pública Odontológica, Facultad de Odontología, Universidad de Concepción, Concepción, Chile.
} 
Se ha estimado que el aporte calórico proveniente del consumo frecuente de papas fritas, completos con mayonesa, sopaipillas, galletas dulces y saladas, chocolates y dulces en general, han pasado a convertirse en parte de la alimentación habitual y puede superar el $40 \%$ de las necesidades energéticas diarias (Olivares et al., 2004; Yáñez et al., 2001). Entre los factores que han contribuido a disminuir el gasto y aumentar la ingesta, se ha señalado la disminución de la actividad física y a un aumento del tiempo destinado a ver televisión (Dietz \& Gortmaker, 1985; Robinson et al., 1993).

Los patrones de actividad física observados en distintas edades son predominantemente de tipo sedentario. Recientemente se ha comunicado que escolares obesas ven televisión más tiempo y realizan actividades menos intensas y más breves que niñas normales. También se ha demostrado en escolares obesos un bajo gasto energético en reposo y reducidos índices de actividad física (Carrasco et al., 1999). En la Región Metropolitana se ha observado que más del $75 \%$ de los adultos realiza menos de 15 minutos de actividad física programada dos veces por semana (Berríos, 1997).

Cada día cobra más importancia la necesidad de disponer información sobre el consumo de alimentos y los patrones alimentarios de la población por su asociación con diferentes enfermedades crónicas no transmisibles. La metodología habitualmente utilizada con este fin es aplicar alguna encuesta alimentaria, aunque ellas son fuertemente cuestionadas por la confiabilidad de las mediciones o por la baja reproducibilidad o precisión de los diversos métodos (Rebolledo, 1998).

Las encuestas más utilizadas en Chile son las que emplean la técnica de interrogatorio, ya sea de Recordatorio de 24 horas (R24H) o de Tendencia de Consumo de Alimentos (Urteaga et al., 2001). Ambas encuestas tienen la ventaja de ser relativamente fáciles de realizar por profesionales entrenados. El método $\mathrm{R} 24 \mathrm{H}$ recoge la información alimentaria del día anterior a la entrevista. Los diversos estudios informan que éste método tiende a subestimar la alimentación del individuo al ser aplicado en una sola oportunidad (Urteaga Ribbeck et al., 2003).

Desde el año 2001 se dispone de nueva recomendaciones energéticas por la $\mathrm{FAO} / \mathrm{OMS}$ las que pueden ser estimadas por fórmulas que consideran edad, sexo y actividad física o utilizando una tabla previamente validada (Aguirre et al., 2010). Están hechas en base al consumo de macronutrientes, dentro de los cuales las principales fuentes de energía de la alimentación provienen de las grasas y los hidratos de carbono. Las proteínas, en cambio, son nutrientes esenciales para la vida, cuyo aporte calórico en 1 gramo es de 4 kilocalorías, siendo su función principal reparar y formar tejidos y no para proporcionar energía (Olivares \& Zacarías, 2010).

Por otro lado, la caries es una enfermedad infecto contagiosa dependiente de la dieta atribuida a la presencia de bacterias orales. La prevalencia y progresión de esta enfermedad se ve influenciada también por factores secundarios que incluyen saliva, flúor e integridad anatómica del esmalte dental (König, 2000). La dieta y la nutrición tienen una influencia directa en la pérdida dental, el mayor componente preventivo tiene relación con la mantención de un equilibrio en la dinámica de desmineralización/ remineralización de la superficie del diente, factor en el cual el calcio es un agente preponderante para fortalecer la calidad de los tejidos dentarios durante su formación (Touger-Decker et al., 2007).

La hipótesis para explicar la relación entre salud oral/enfermedad con la salud general incluye los efectos de la enfermedad oral en la inflamación (Taylor et al., 2006) y los efectos de la pérdida dental y una pobre función masticatoria sobre la dieta y estado nutricional. Por lo tanto, una pobre salud y función oral tienen implicancia como factores de riesgo de una dieta pobre (De Marchi et al., 2008).

Para registrar la cantidad de caries existente se utiliza el índice epidemiológico COPD. El componente $\mathrm{C}$ describe el número de dientes afectados por caries. El componente $\mathrm{O}$ señala el número de dientes obturados a consecuencia de caries, cuyas obturaciones se encuentran en óptimas condiciones y el $\mathrm{P}$, indica el número de dientes perdidos por caries o con indicación de extracción debido a caries. EI COPD es el resultado de la suma de estos valores (OMS, 1997).

Con el propósito de aportar mayores antecedentes para planificar estrategias de intervención que fomenten un estilo de vida saludable, se desarrolló el presente estudio cuyo objetivo es describir los patrones alimentarios, actividad física e historial de caries según condición urbano/rural de losadolescentes de 17 años pertenecientes a la población educacional de Florida. 


\section{MATERIAL Y MÉTODO}

Este es un estudio observacional descriptivo de diseño transversal cuya población de análisis corresponde a la totalidad de los alumnos pertenecientes al Liceo Luis de Alava y al Colegio Inés Enríquez Frodden, que cumplieron 17 años antes del 31 de Mayo y que sean de la comuna de Florida. Cumplieron los requisitos de inclusión y estuvieron dispuestos a participar 86 jóvenes, cuyos padres/apoderados otorgaron el consentimiento informado. Se procedió a recolectar la información por medio de 2 etapas: la primera realizada por un observador calibrado, mediante un examen clínico de la cavidad oral con espejo, sonda, guantes, mascarilla y luz artificial proporcionada por el box dental y una entrevista de información básica, origen y nivel de actividad física realizada por un segundo observador. Los datos se registraron en una ficha especialmente diseñada para el caso. La segunda etapa fue realizada en el box de nutrición para determinar el índice de masa corporal (IMC), estado nutricional y entrevista $\mathrm{R} 24 \mathrm{H}$ efectuada por un tercer observador y aplicada en tres oportunidades, para aumentar la confiabilidad de los datos obtenidos; además se cuantificó el peso y la talla de cada persona usando una balanza marca Seca, cuya escala presenta una sensibilidad de 0,1 kilogramos y tallímetro Seca cuya escala presenta una sensibilidad de $1 \mathrm{~mm}$, que fue la misma para todos y ambas pertenecientes al Hospital de Florida, obteniendo uniformidad en los resultados obtenidos.

Para determinar los valores de kilocalorías y macronutrientes consumidos diariamente, se utilizó una lista tabulada de nutrición en formato Excel, que determinó los valores nutricionales de cada porción de alimento y de cada individuo en el transcurso del día. Se considera relevante medir las calorías y su distribución por persona por lo cual se transformaron los gramos de macronutrientes en Kcal. Para simplificar la variable estado nutricional se unieron las categorías Sobrepeso y Obeso en uno sólo, denominado Obeso.

\section{RESULTADOS}

Tabla II. Kcal consumidas y recomendadas para nivel de Actividad Física, según sexo. Florida año 2011.

\begin{tabular}{lccccccccc}
\hline & Sedentario & DE & Recomendado & Moderado & DE & Recomendado & Activo & DE & Recomendado \\
\hline Hombre & 3014 & 781 & 2200 & 2791 & 825 & $2400-2800$ & 3130 & 997 & $2800-3200$ \\
Mujer & 2152 & 622 & 1800 & 1992 & 439 & 2000 & 2169 & 342 & 2400 \\
\hline
\end{tabular}

La distribución de la población de estudio junto con las kilocalorias consumidas se señalan en las Tablas I y II . Se observa, además, que los jovenes que realizan actividad física (moderada y activa) consumen una cantidad de energía cercana a la recomendada. A diferencia de esto, los sedentarios que presentan ausencia absoluta de actividad física, tienen una ingesta calórica mayor a lo recomendado. Esta diferencia se acentúa al comparar por sexo, donde la diferencia es más marcada en hombres que en mujeres sedentarias.

Tabla I. Distribución de la población de estudio según sexo y condición Urbano/rural. Florida año 2011.

\begin{tabular}{lccc}
\hline & Rural & Urbano & Total \\
\hline Masculino & 29 & 18 & 47 \\
Femenino & 14 & 25 & 39 \\
Total & 43 & 43 & $\mathbf{8 6}$ \\
\hline
\end{tabular}

La mayor concentración de hombres obesos se encuentra en el sector rural, con un $27,4 \%$ de los hombres totales en estudio contra $8,5 \%$ en los urbanos. En el caso de las mujeres ocurre lo contrario, donde un $35,8 \%$ de las obesas se concentra en el sector urbano contra $12,8 \%$ en las rurales con respecto a la totalidad de las mujeres en estudio (Fig. 1).

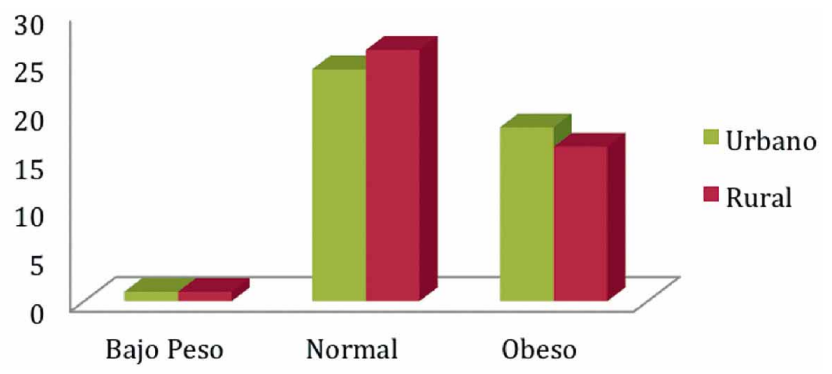

Fig. 1. Distribución Estado Nutricional según origen, Florida año 2011.

La cantidad consumida corresponde a un 33,5\% de la ingesta diaria recomendada. La diferencia se acentúa aún más para las mujeres donde consumen sólo el 26,8\% (Fig. 2).

No se encontraron diferencias estadística-mente significativas $(p>0.05)$ al comparar COPD entre adolescentes de peso normal y obesos, siendo en ambos casos Muy alto y se señalan en la Tabla III. 


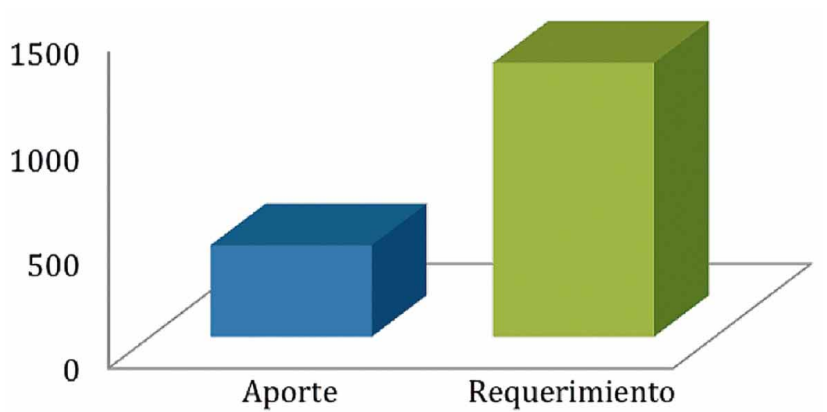

Fig. 2. Consumo de Calcio en jóvenes adolescentes, Florida año 2011.

Tabla III. Distribución del Índice COPD según sexo y estado nutricional. , Florida año 2011.

\begin{tabular}{lcccc}
\hline & Bajo peso & Normal & Obeso & Media \\
\hline Masculino & 11,5 & 8,77 & 8,73 & $\mathbf{8 , 8 7}$ \\
Femenino & - & 9,5 & 9,05 & $\mathbf{9 , 2 8}$ \\
Media & $\mathbf{1 1 , 5}$ & $\mathbf{9 , 0 6}$ & $\mathbf{8 , 9 1}$ & $\mathbf{9 , 0 6}$ \\
\hline
\end{tabular}

\section{DISCUSIÓN}

En el presente estudio se encontró, de acuerdo al estado nutricional, un $2 \%$ de los jóvenes con bajo peso, un $58 \%$ normales y un $40 \%$ obesos, lo que revela la mala condición nutricional de los adolescentes de Florida. Esto se correlaciona con datos entregados en la Encuesta Nacional de Salud (ENS) del MINSAL para el año 2009-2010 (MINSAL, 2010; Olivares \& Zacarías), que indica que más de un $40 \%$ de la población se encuentra con sobrepeso y/u obesidad.

En las mujeres casi el $50 \%$ pertenece a la categoría de obesidad, en cambio en los hombres se observó una predominio de normalidad, según su estado nutricional, con un $15 \%$ de obesidad, esto se explica debido a que las mujeres, si bien consumieron en su dieta en promedio $79,24 \mathrm{Kcal}$ más de lo recomendado y los hombres $271,3 \mathrm{Kcal}$, son éstos quienes realizan mayor actividad física. Esto se fundamenta, al igual que en otros artículos (Reyes et al. 2011), en que el problema asociado a la obesidad en el adolescente, es el consumo energético dentro de límites normales, pero relacionado a un insuficiente gasto energético total, por lo tanto se observa que el principal determinante de la retención calórica y por ende de la obesidad, sería el bajo gasto energético.
Las cantidades de calcio ingeridas por los estudiados son el $33,5 \%(435,41 \mathrm{mg} /$ día $)$ del consumo diario recomendado (1300 mg/día) (Olivares et al.) siendo esto muy inferior a lo establecido, con una diferencia de $864,59 \mathrm{mg}$. A pesar de que ambos sexos se encontraron en niveles inferiores a la norma, la diferencia fue aún más pronunciada en las mujeres, quienes tuvieron un $26,8 \%(341,69$ $\mathrm{mg} / \mathrm{día}$ ) del consumo recomendado para ellas. Similares resultados son encontrados en otros estudios, donde informan que el adolescente frecuentemente disminuye la ingesta de lácteos, llegando alrededor de $480 \mathrm{mg} /$ día, aumentando el consumo de bebidas gaseosas, café, té y alcohol (Olivares et al., ENS). Lo anterior es de importancia, porque estos adolescentes se encuentran aún durante su desarrollo óseo y el consumo de calcio, junto con el nivel de actividad física, son los principales determinantes ambientales de la ganancia de masa ósea máxima, evitando fracturas y enfermedades asociadas como osteoporosis (Reyes et al.).

La creciente y determinante inactividad física de los adolescentes podría inducir cambios en las normas nutricionales, adecuándolas a un pobre desarrollo de actividad física, sin embargo, sería importante lograr estrategias nacionales de fomento en la actividad física, permitiendo un balance energético y ganancia muscular, recordando siempre que un músculo esquelético desarrollado permite una correcta metabilización de los nutrientes ingeridos y de las grasas corporales acumuladas.

En cuanto al COPD se observó que no existió una diferencia estadísticamente significativa entre sexo, estado nutricional, ni tampoco en el origen, siendo en todos los casos clasificado el COPD según la Organización Mundial de la Salud (OMS, 1997) como Muy Alto. Cabe destacar que al asociar la cantidad de calcio consumido versus el índice de historia de caries COPD, no existió una tendencia marcada en su distribución que permita demostrar una relación entre ambos factores. Es importante recalcar que el estudio es de tipo transversal, y por ende no necesariamente las cantidades de calcio ingeridas actualmente tienen relación alguna con las consumidas en etapas más tempranas de su formación, donde el calcio cobra mayor relevancia, asociado a edades de desarrollo temprano del germen dentario. 


\section{CONCLUSIONES}

Existe un alto consumo de productos con bajo índice nutricional, hipercalórico e incluso dañino para la salud, debido a sus bajos precios y alta accesibilidad. Asimismo, la creciente cantidad de productos de preparación rápida y fácil, con bajo valor nutricional y altos índices de grasas saturadas e hidratos de carbono, contribuyen a los altos valores de obesidad. Además, en su gran mayoría son consumidos con mayor frecuencia y cantidad en la tarde o noche, donde el metabolismo basal humano se encuentra disminuido.

Ya no existen marcadas diferencias entre la alimentación de la gente de la zona rural y urbana, debido a la adopción de características y costumbres similares, acompañado a la mayor facilidad de transporte y comunicación del campo con la ciudad. Se ha producido una disminución del consumo de productos de elaboración propia (leche, queso, pan amasado, vegetales, frutas) que han equilibrado las balanzas alimenticias hacia dietas menos nutritivas.

Este estudio permite reflejar, que el adolescente presenta un marcado desorden alimentario, que si bien se encuentra dentro de los limites energéticos de ingesta alimentaria, lo hace acompañado de niveles de actividad física bajos y con un subconsumo de nutrientes especialmente de calcio; es por ello que las políticas y estrategias nacionales deberían apuntar, además de reforzar la alimentación saludable, a estilos de vida saludables y menos sedentarios.

Con respecto al índice COPD, se evidencia un estado dental deteriorado en toda la población, lo que requiere de un análisis más profundo en estudios posteriores que intenten identificar factores críticos como cepillos, sedas, pastas y visitas regulares al dentista.

ARAYA, V. C.; MARCHANT, O. D.; RIVAS, D. P.; PALMA, T. C. \& POBLETE, F. O. Nutritional status of 17-year-old adolescents and its relation with caries history. Florida 2011. Int. J. Odontostomat., 8(2):171-176, 2014.

SUMMARY: Given the high rates of childhood obesity, it is necessary to collect current information to guide new prevention programs in earlier stages of adolescent development. The objective of the present study is to evaluate the nutritional status of 17-year-old adolescents of educational establishments in Florida, eighth region, Chile, and its relation with dental caries prevalence in permanent dentition (COPD). A cross-sectional descriptive study was carried out in a sample of 86 students, using a previously validated survey, about the dietary intake of the last 24 hours, which was applied three times; weight and height were also considered. During an interview information was obtained about sex, rural and urban background. Finally a dental examination of the history of decay was applied (COPD index, in Spanish). Test materials used were masks, latex gloves, and stadiometer calibrated weight and height rod. According to the nutritional status, $2 \%$ of adolescents were underweight, $58 \%$ had a normal weight and $40 \%$ were obese, the rate of obesity is higher in women in proportion to men, where nearly $50 \%$ of cases were in obese condition and were statistically significant. Moreover, a homogeneous distribution was observed, regardless of where they came from (rural or urban conditions). In relation to COPD index, an 8.87 in male and 9.28 in female were obtained with no significant differences between men and women. In addition, there was no statistical significance on COPD in relation to the nutritional status. It was observed that 17-year-old adolescents from Florida's educational establishments present a nutritional deficit status reflected in high obese percentages, which according to $\mathrm{WHO}$, was also related to a significant history of decay.

KEY WORDS: adolescents, caries history, nutrition.

\section{REFERENCIAS BIBLIOGRÁFICAS}

Aguirre, C. M. L.; Castillo, D. C. \& Le Roy, O. C. Desafíos Emergentes en la Nutrición del Adolescente. Rev. Chil. Pediatr., 81(6):488-97, 2010.

Berríos, $\mathrm{X}$. Time trends in risk factors for chronic diseases: is a new epidemic coming? Rev. Med. Chil., 125(11):1405-7, 1997.

Carrasco, N. F.; Reyes S. E.; Charlin De Groote, V. \& Kehr, S. J. Gasto energético de reposo medido versus gasto energético estimado por fórmula en niños obesos. Rev. Chil. Pediatr., 70(2):107-12, 1999.
De Marchi, R. J.; Hugo, F. N.; Hilgert, J. B. \& Padilha, D. M. Association between oral health status and nutritional status in south Brazilian independent-living older people. 0:546-53, 2008.

Dietz, W. H. Jr. \& Gortmaker, S. L. Do we fatten our children at the television set? Obesity and television viewing in children and adolescents. Pediatrics, 75(5):807-12, 1985.

Gidding, S. S.; Dennison, B. A.; Birch, L. L.; Daniels, S. R.; Gilman, M. W.; Lichtenstein, A. H.; Rattay, K. T.; Steinberger, J.; Stettler, N.; Van Horn, L. \& American 
Heart Association. Dietary recommendations for children and adolescents: a guide for practitioners. Pediatrics, 117(2):544-59, 2006.

König, K. G. Diet and oral health. Int. Dent. J., 50(3):162-74, 2000.

Larson, N. I.; Story, M.; Eisenberg, M. E. \& Neumark-Sztainer, D. Food preparation and purchasing roles among adolescents: associations with sociodemographic characteristics and diet quality. J. Am. Diet Assoc., 106(2):211-8, 2006.

Niemeier, H. M.; Raynor, H. A.; Lloyd-Richardson, E. E.; Rogers, M. L. \& Wing, R. R. Fast food consumption and breakfast skipping: predictors of weight gain from adolescence to adulthood in a nationally representative sample. J. Adolesc. Health, 39(6):842-9, 2006.

Ministerio de Salud (MINSAL). ENS - Encuesta Nacional de Salud 2009-2010. Gobierno de Chile, 2010. Disponible en http://www.redsalud.gov.cl/portal/url/item/ 99bbf09a908d3eb8e04001011 f014b49.pdf

Olivares, S.; Kain, J.; Lera, L.; Pizarro, F.; Vio, F. \& Morón, C. Nutritional status, food consumption and physical activity among Chilean school children: adescriptive study. Eur. J. Clin. Nutr., 58(9):1278-85, 2004.

Olivares, S. \& Zacarías, I. Guía de alimentación saludable y necesidades nutricionales del adulto. Instituto de nutrición y tecnología de los alimentes, Universidad de Chile, 2010. Disponible en http://www.inta.cl/ material_educativo/cd/3GuiAli.pdf

Organización Mundial de la Salud (OMS). Encuestas de Salud Buco Dental, Métodos Básicos. $4^{a}$ ed. Ginebra, Organización Mundial de la Salud, 1997.

Rebolledo, A. Encuestas Alimentarias. Rev. Chil. Nutr., 25(1):28-34, 1998.

Reyes, J. M.; Díaz, B. E.; Lera, M. L. \& Burrows, A. R. Intake and energy metabolism in a sample of overweight and obese Chilean adolescents. Rev. Med. Chil., 139(4):42531, 2011.

Robinson, T. N.; Hammer, L. D.; Killen, J. D.; Kraemer, H. C.; Wilson, D. M.; Hayward, C. \& Taylor, C. B. Does television viewing increase obesity and reduce physical activity? Cross-sectional and longitudinal analyses among adolescent girls. Pediatrics, 91(2):273-80, 1993.

Taylor, B. A.; Tofler, G. H.; Carey, H. M.; Morel-Kopp, M. C.; Philcox, S.; Carter, T. R.; Elliott, M. J.; Kull, A. D.; Ward, C. \& Schenck, K. Full-mouth tooth extraction lowers systemic inflammatory and thrombotic markers of cardiovascular risk. J. Dent. Res., 85(1):74-8, 2006.
Touger-Decker, R.; Mobley, C. C. \& American Dietetic Association. Position of the American Dietetic Association: oral health and nutrition. J. Am. Diet. Assoc., 107(8):1418-28, 2007.

Urteaga Ribbeck, C.; Pinheiro Fernandes, A. C. \& Atalah Samur, E. Comparison of results from two dietary interview methods. Arch. Latinoam. Nutr., 53(2):172-7, 2003.

Urteaga, C.; Ramos, R. I. \& Atalah, E. Validation of global nutrition assessment in elders. Rev. Med. Chil., 129(8):871-6, 2001

Yáñez, R.; Olivares, S.; Torres, I.; Guevara, M. \& Díaz, N. Consumo de alimentos en escolares chilenos. Su relación con las guías y la pirámide alimentaria. Rev. Chil. Nutr., 28(3):422-8, 2001.

Dirección para Correspondencia:

Dr. Carlos Araya Vallespir

Avenida Roosevelt 1550

Concepción

CHILE

Email: carlos.araya@udec.cl

Recibido: 26-06-2013

Aceptado: 02-05-2014 